\title{
Study of One Kind of Extended Proportional Guidance Law
}

\author{
Yao Jun, Feng Hai-tao and Sun Peng \\ Shenyang Ligong University, Shenyang 110159, China \\ yaojunjun@sina.com,sylaizh@163.com
}

\begin{abstract}
One kind of extended proportional guidance law is presented. This guidance law can be used without restriction about the motion speed of missile and target, and this is a different point with the classical proportional guidance law. In the case of random alter the tracking target curvature radius, compared with classical proportional guidance law under the proper random quantity, the normal overload of trajectory is relatively small, and has certain advantage, so the study has significant reference value in actual application. And interception time and normal overload have good performance. Tracking performance for high maneuverability target is better than the classical proportional guidance law. A target intercepting simulation based on MATLAB of the proportional coefficients and line-of-sight angular velocities is generated to analyze the trajectory properties. The effect of guidance coefficients on trajectory properties is studied. Through simulation, we simplify the problem by ignoring the actual factors so that a certain level of error is expected. The situation in practical requires more in-depth analysis and research.
\end{abstract}

Keywords: Extended Proportional Guidance Law; Maneuvering target; Orbital transfer motion; Normal overload

\section{Introduction}

Traditional proportional guidance law is defined that the los rate is in proportion to tiltangle of missile trajectory. The command acceleration is applied at vertical sight. We get this optimal control guidance law on the condition that assuming the velocity of missile and target is a constant and low maneuverability target [1-4].But to the intercepting maneuverable targets, the traditional proportional guidance law is unsatisfying and overload demand of acceleration command is not low enough. In order to adapt to the complex combat circumstance, the development and application of a new guidance law which is adapt to intercept high maneuverability target is a urgent task [5-8]. In this paper an extended proportional guidance law is designed based on the traditional dynamic differential equation, and this improved performance is for maneuvering targets, analyzing the affect of normal overload and trajectory curvature by extended proportional guidance law.

\section{Construct Relative Motion Equation of Extended Proportional Guidance Law}

In the result of some development, the differential equations [9-11]

$$
\ddot{r}+f \dot{r}+g r=0
$$


is used to describe the elliptical trace of astronomical under eccentric pressure effect, $f$ is the scalar function $r$ and $g$ is the scalar function $\theta$ in the polar coordinates of ellipse plane. For planar motion, $f=-(i / l)$, and $i=\ddot{r}^{2} \theta$ is angular momentum, introducing $i$ and $f$ into Eq.(1) leads to

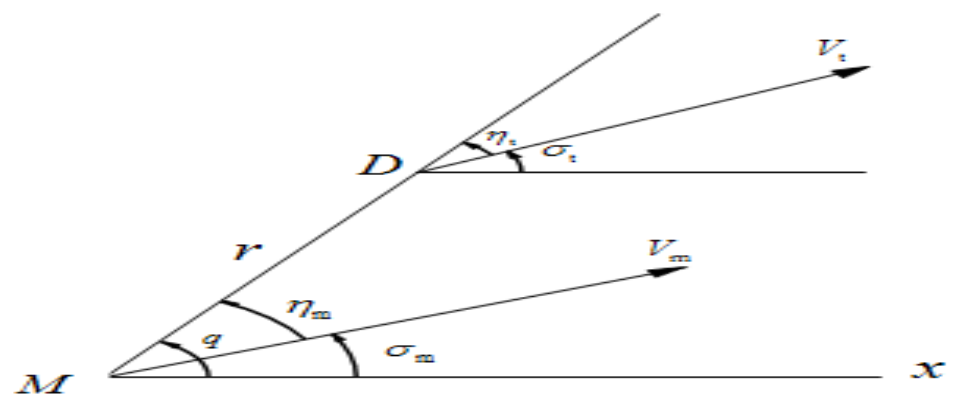

Figure 1. Geometric Position Relation between Missile and Target

$$
\frac{d}{d t}\left(\frac{\dot{r}}{l}\right)=-\frac{g}{l} r
$$

Assuming on one time, target at point $\mathrm{D}$, missile is location $\mathrm{M}$, relative motion as the Figure 1.The DM is the target line $r$. Separating the relative velocity of point $M$ into target line and normal, the Eq.(2) can be converted [12-13]to

$$
\left\{\begin{array}{l}
\frac{d}{d t}\left(\frac{v_{\mathrm{r}}}{l}\right)-\frac{\dot{q}}{l} v_{\mathrm{q}}=-\frac{g}{l} r \\
\frac{d}{d t}\left(\frac{v_{\mathrm{q}}}{l}\right)+\frac{\dot{q}}{l} v_{\mathrm{r}}=0
\end{array}\right.
$$

Where $v_{\mathrm{r}}=\dot{r}$ is the radial component of relative velocity about point $M$, and $v_{\mathrm{q}}=r \dot{q}$ is the normal component. From the following equation

then

$$
\frac{\dot{r}}{\dot{q}}=\frac{d r}{d q} \text { divided by } q,
$$

We get

$$
\dot{q}^{2} \cdot \frac{d r}{d q}=\ddot{r}-\ddot{q} \cdot \frac{d r}{d q}
$$

Introducing $v_{\mathrm{r}}=\dot{r}$ and $v_{\mathrm{q}}=r \dot{q}$ into Eq.(3) leads to the acceleration equation about target line and normal

$$
\ddot{r}-r \dot{q}^{2}=-g r+(\dot{l} / l) \cdot r \quad \text { (5) } \quad r \ddot{q}+2 \dot{r} \dot{q}=(l / l) \cdot q
$$

From the Eq.(5) and (6),we can get

$$
\frac{g r^{2}}{\dot{q}^{2}}=r^{2}+2\left(\frac{d r}{d q}\right)^{2}-r \cdot \frac{\ddot{r}-\ddot{q}(d r / d q)}{\dot{q}^{2}}
$$

Introducing Eq.(4) into Eq.(7) leads to 


$$
\frac{g r^{2}}{\dot{q}^{2}}=r^{2}+2\left(\frac{d r}{d q}\right)^{2}-r \cdot \frac{d^{2} r}{d q^{2}}
$$

By defining $v_{\mathrm{m}}$ is relative velocity of point $M, \sigma_{\mathrm{m}}$ is the angle between $v_{\mathrm{m}}$ and x-axis, the component in rectangular coordinate system can be written as $\dot{x}=v_{\mathrm{m}} \cos \sigma_{\mathrm{m}}, \dot{y}=v_{\mathrm{m}} \sin \sigma_{\mathrm{m}}$, take $x=r \cos q$ and $y=r \sin q$ derivation of time leads to

$$
\begin{aligned}
& v_{\mathrm{m}} \cos \sigma_{\mathrm{m}}=\dot{r} \cos q-r \dot{q} \sin q \\
& v_{\mathrm{m}} \sin \sigma_{\mathrm{m}}=\dot{r} \sin q+r \dot{q} \cos q
\end{aligned}
$$

and $\sigma_{m}$ could be calculated by

$$
\tan \sigma_{\mathrm{m}}=\frac{\dot{r} \sin q+r \dot{q} \cos q}{\dot{r} \cos q-r \dot{q} \sin q}
$$

Numerator and denominator of above formula are divided by $\dot{q}$, then the result derivation of $q$, we have

$$
\frac{1}{\cos ^{2} \sigma_{\mathrm{m}}} \frac{d r}{d q}=\frac{r^{2}+2\left(\frac{d r}{d q}\right)-r\left(\frac{d^{2} r}{d q^{2}}\right)}{\left[\left(\frac{d r}{d q}\right) \cos q-r \sin q\right]^{2}}
$$

and $\frac{1}{\cos ^{2} \sigma_{\mathrm{m}}}=\tan ^{2} \sigma_{\mathrm{m}}+1=\frac{r^{2}+\left(\frac{d r}{d q}\right)^{2}}{\left[\left(\frac{d r}{d q}\right) \cos q-r \sin q\right]^{2}}$,

then

$$
\frac{d r}{d q}=\frac{r^{2}+2\left(\frac{d r}{d q}\right)-r\left(\frac{d^{2} r}{d q^{2}}\right)}{r^{2}+\left(\frac{d r}{d q}\right)^{2}}
$$

In Figure $1, \eta_{\mathrm{m}}$ is angle between the relative speed $v_{\mathrm{m}}$ and DM line. We can get $\eta_{\mathrm{m}}=q-\sigma_{\mathrm{m}}$, and, $\operatorname{ctg} \eta_{\mathrm{m}}=\frac{\dot{r}}{r \dot{q}}=\frac{d r}{r d q}$

then we have

$$
\sin ^{2} \eta_{\mathrm{m}}=\frac{r^{2}}{r^{2}+(d r / d q)^{2}}
$$

Bringing Eq.(12) and Eq.(8) into Eq.(11) leads to

$$
\frac{\dot{\sigma}_{\mathrm{m}}}{\dot{q}}=\frac{g}{\dot{q}^{2} \sin ^{2} \eta_{\mathrm{m}}}
$$

We get

$$
\frac{g}{l} r=\frac{\dot{q}}{p} \text { and } p=r-\varepsilon x \text {, or }
$$




$$
g=\frac{r \dot{q}^{2}}{p}
$$

Introducing Eq.(14) into Eq.(13) leads to

$$
\frac{\dot{\sigma}_{\mathrm{m}}}{\dot{q}}=\frac{g}{\dot{q}^{2} \sin ^{2} \eta_{\mathrm{m}}}=\frac{r}{p \sin ^{2} \eta_{\mathrm{m}}}
$$

From engineering considerations, we can ignore the data $\varepsilon$, and data $r$ use $p$ instead.

Then Eq.(15) can be rewritten as

$$
\frac{\dot{\sigma}_{\mathrm{m}}}{\dot{q}}=\frac{1}{\sin ^{2} \eta_{\mathrm{m}}}
$$

Eq.(16) is a guidance law which base on the traditional dynamic differential equations about angular velocity of missile relative velocity and line-of-sight target. This formula is called extended proportional guidance law. This guidance law can be used without restriction about the motion of missile and target, and it should be allowed a missile using the guidance law to attack any high speed and maneuvering target in theory. But the classical proportional guidance law cannot reach.

\section{Simulative and Analyses Orbital Transfer Motion of Target}

In the procedure of simulation, to take target after perception of danger distance on the vertical plane taking sinusoidal transfer motion for an example, use the MATLAB tools to get the trajectory simulation of the extended proportional guidance law, taking simulative parameters: missile velocity is $V_{m}=1000 \mathrm{~m} / \mathrm{s}$, target velocity is $800 \mathrm{~m} / \mathrm{s}$, target angel of course is $45^{\circ}$, initial coordinates of target is $(0,10000)$, units are all $\mathrm{m}$. The amplitude of the sinusoidal transfer motion for target is $400 \mathrm{~m}$, and cycle of the sinusoidal transfer motion is $T=\pi s$, and rotational speed of the sinusoidal transfer motion is $\omega=2 \pi / T(\mathrm{rad} / \mathrm{s})$. Curve of pursuit curve is following the Figure 2. We can see that trajectory can maintain the stability and smooth, and it has little curve at the beginning of trajectory. But trajectory has no big fluctuation and maintains stability and smooth as transfer motion of missile by perception of target.

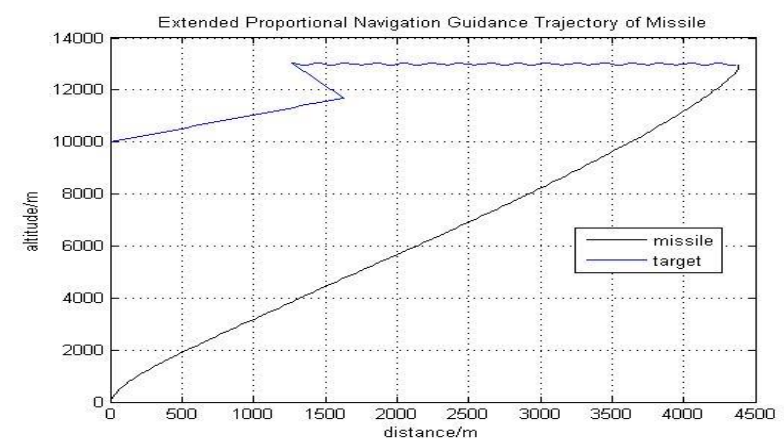

Figure 2. Trajectory with Target Random take Sinusoidal Transfer Motion $(A=400)$

In Figure 3, when the magnitude of target transfer motion is changed to $500 \mathrm{~m}$, compared with Figure 2, the winding level of trajectory front is larger. And it also leads to larger normal overload, but trajectory doesn't have changed so much with the distance making short when 
the target takes transfer motion. Tracking the target accurately by the extended proportional guidance law, but the classical proportional guidance law does not have this advantage.

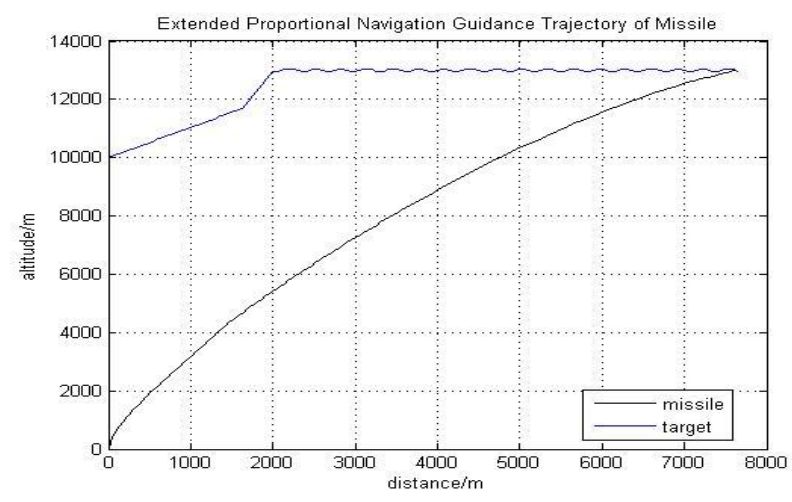

\section{Figure 3. Trajectory with Target Random take Sinusoidal Transfer Motion $(A=500)$}

The Figure 4 is the function of extended proportional guidance law after perception of danger distance, and it is tending towards stability before transfer motion. So we can get that the target can adjust itself follow the target transfer motion when the target takes sinusoidal orbital transfer motion using extended proportional guidance law. It can be corrected itself follow the trajectory angle and target line angle alteration.

Figure 5 is the normal overload curve of taking sinusoidal orbital transfer motion as target perception of danger distance, because the initial configuration result in the normal overload is instable at the initial stage, as the time goes by tracking the target, the normal overload tends to zero gradually. In the case of short distance between target and missile, normal overload is also tends to stable. And it isn't affected by transfer motion of target, so it may attack the target effectively and not be affected by the transfer motion of target. Finally we can get the right normal overload under the adjustment of itself.

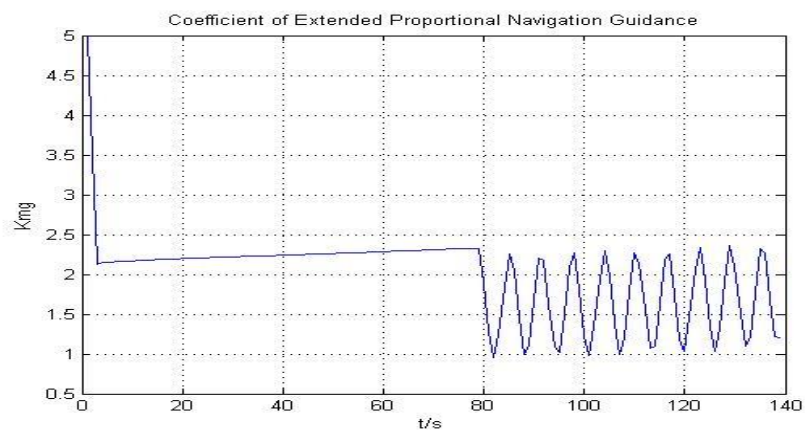

\section{Figure 4. Extended Proportional Guidance Law Coefficient by Taking Sinusoidal Transfer Motion}

Figure 6 shows the comparison of trajectory tracks of extended proportional guidance law and classical proportional guidance law when target random takes sinusoidal transfer motion with perception of danger distance $5000 \mathrm{~m}$, and the amplitude of the sinusoidal transfer motion for target is $\mathrm{A}=400 \mathrm{~m}$. We may assume the velocity of missile $V_{\mathrm{m}}=800 \mathrm{~m} / \mathrm{s}$, the 
velocity of target $V_{\mathrm{t}}=420 \mathrm{~m} / \mathrm{s}$. It can be seen from the Figure 6 . When trajectories maintain doesn't have perception of transfer motion, the target could not track wonderful by the classical proportional guidance law after perception of transfer motion. As the trajectory fluctuates, bending level of trajectory enlarges when the distance between target and missile is shorten. The extended proportional guidance law has good track performance, and trajectories are smoothly and stably.

Changing the velocity of missile is $V_{\mathrm{m}}=1000 \mathrm{~m} / \mathrm{s}$, and the velocity of target is $V_{\mathrm{t}}=600 \mathrm{~m} / \mathrm{s}$, and the amplitude of the sinusoidal transfer motion for target is also $400 \mathrm{~m}$. Figure $6(\mathrm{~b})$ is the tracking trajectory of extended proportional guidance law and classical proportional guidance law. We can know that the advantage of classical proportional guidance law is time shortage for attack on target. It results in instable system by enlarge bending level of trajectory suddenly.

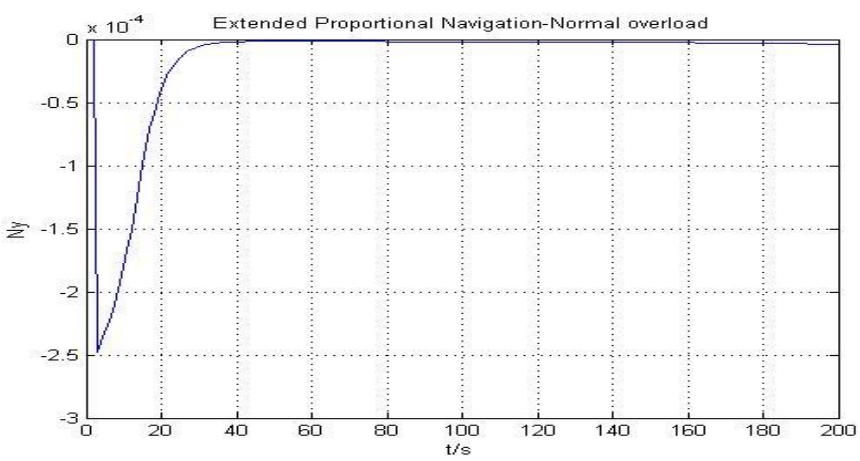

Figure 5. Normal Overload Coefficient by taking Sinusoidal Transfer Motion
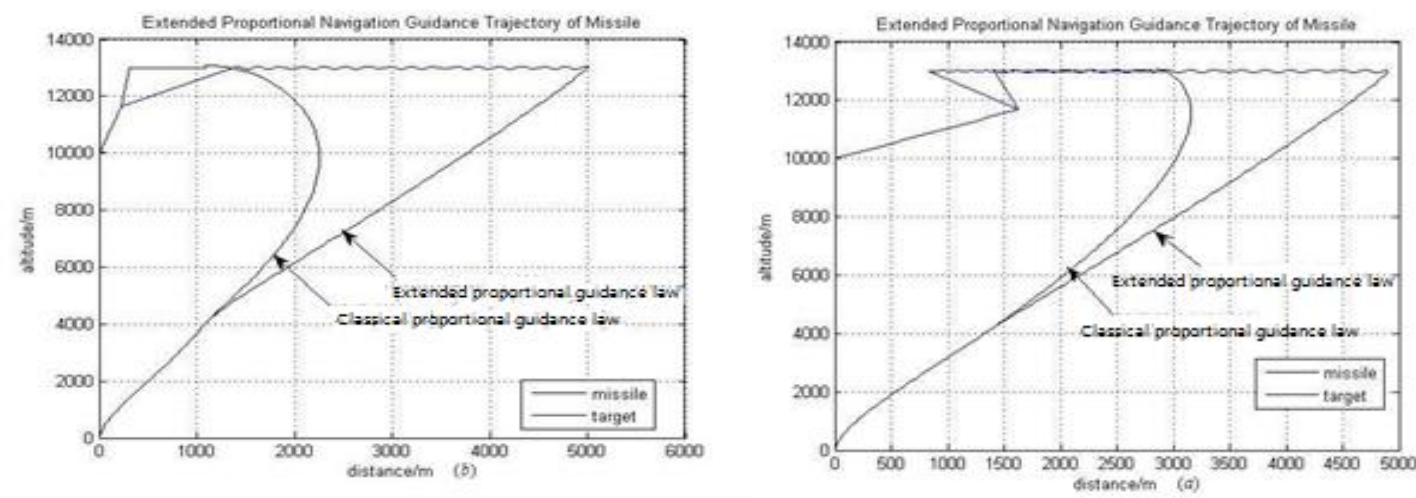

Figure 6. Comparison about Trajectory with Target Random take Sinusoidal Transfer Motion $(A=400)$

\section{Relative Motion Equation}

The relative motion equation between the Missile and target can be written as 


$$
\begin{aligned}
& \frac{\mathrm{d} r}{\mathrm{~d} t}=V_{T} \cos \eta_{T}-V \cos \eta \\
& r \frac{\mathrm{d} q}{\mathrm{~d} t}=V \sin \eta-V_{T} \sin \eta_{T} \\
& q=\sigma+\eta \\
& q=\sigma_{T}+\eta_{T} \\
& \frac{\mathrm{d} \sigma}{\mathrm{d} t}=K \frac{\mathrm{d} q}{\mathrm{~d} t}
\end{aligned}
$$

With the given function of $V$ (missile speed), $V_{T}$ (target speed), $\sigma_{T}$, as well as the initial conditions of one of $r_{0}, q_{0}, \sigma_{0}$ or $\eta_{0}$, equation (17) can be solved by graphical method or numerical integration method. We can only get analytical solutions of the equation (17) under special condition (when $K=2$, the target is traveling in constant velocity so as the missile).

\section{A Trajectory Properties}

1) Straight trajectory: the initial condition is $\dot{\sigma}=0$, therefore $\dot{q}=0, \dot{\eta}=0$, thus $\eta=\eta_{0}=$ constant. The second equation of equations (17), which is the condition of travelling along a straight trajectory, can be resolve as

$$
V \sin \eta-V_{T} \sin \eta_{T}=0
$$

Equation (18) indicates the velocity vector of the missile equals to the velocity vector of the target in the direction, which is perpendicular to the target line. The relative velocity always point to the target[8]. So in order to follow a straight trajectory, the pre-angle $\eta_{0}$ of the velocity vector of the missile should strictly meet

$$
\eta_{0}=\left.\arcsin \left(\frac{V_{T}}{V} \sin \eta_{T}\right)\right|_{t=t_{0}}
$$

When $\dot{q}=0^{\circ}$ and $\dot{q}=180^{\circ}$, the condition meet the equation (18), the trajectory is a straight line. When transmitting to other directions, the condition does not meet equation (18), which is $\dot{q} \neq 0$, and then the target line is continuously rotating in the guidance process, thus • $\sigma \neq 0$, which also indicates the absolute trajectory is not a straight line, and the relative trajectory is not a straight line as well. The q value changes slightly in the guidance process. If the emission directions are the same (the $q_{0}$ values are the same), even though the relative distances $r_{0}$ are different in the initial condition, $q_{K}$ (the target line angle when the missile hit the target) remain the same and target line angle of the target also remain the same, thus $q_{K}$ and $r_{0}$ are independent. The conclusions above can be proved as following: when the missile hits the target successfully $r_{K}=0$, according to the second equation of equations (18), we can get

$$
\begin{gathered}
\eta_{K}=\arcsin \left[\frac{1}{p} \sin \left(q_{K}-\sigma_{T K}\right)\right] \\
\eta_{K}=\eta_{0}+(1-K)\left(q_{K}-q_{0}\right)
\end{gathered}
$$


Substitute the equation (21) into equation (19), and put the initial condition $\eta_{0}=0^{\circ}$ (direct targeting emission) and $\eta_{0}=0^{\circ}$ into equation (19), then we get

$$
q_{K}=q_{0}-\frac{1}{K-1} \arcsin \left(\frac{\sin q_{K}}{p}\right)
$$

According to the equation above, we can get that the target line angle $q_{K}$ and $r_{0}$ are independent. Since $\sin q_{K} \leq 1$, then

$$
\left|q_{K}-q_{0}\right| \leq \frac{1}{K-1} \arcsin \left(\frac{1}{p}\right)
$$

2) Launching to other directions: the maximum value of the target line rotation $\left|q_{K}-q_{0}\right|_{\max }$ is represented by $\Delta q_{\max } p$ is the ratio of missile velocity and target velocity. We set $K=5$ and $p=2$, then substitute them into equation (22), and we can get $\Delta q_{\max }=7.5^{\circ}$

This result corresponds to the case of $q_{0}=97.5^{\circ}$ and $q_{K}=90^{\circ}$. In practice, the turned angle of the target line is less than $\Delta q_{\max }$. When $q_{0}=33.7^{\circ}$ and $q_{K}=30^{\circ}$, the turned angle of target line is $3.7^{\circ}$. The value of $\Delta q_{\max }$ is determined by the coefficient $K$ and the velocity ratio $p$. The variation trend is shown in Figure 7. The curves from the top to bottom in Figure 7represent the relationship between the maximum rotation angle and the $\mathrm{K}$ value when $p=$ $1.5, p=2, p=3$ and $p=4$. According to the Figure 7, the maximum rotation angle of the target line is decreasing with the increasing velocity ratio $p$, and the increasing coefficient $K$.

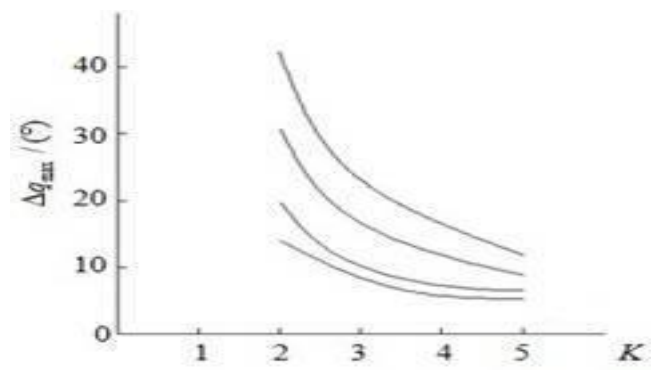

\section{Figure 7. Maximum Rotation Angle of the Target Line $\left(\eta_{0}=0^{\circ}\right)$}

3) Necessary normal over-load: If $\dot{\sigma}$ is the turning velocity of the missile and $\dot{q}$ is the rotation angular velocity of the target line, $\dot{\sigma}$ is proportional to $\dot{q}$ in proportional navigation guidance law. Thus the necessary normal over-load is proportional to the rotation angular velocity of the target line.

Take the derivative of the second equation in equations (17) in terms of time on both sides. We get

$$
\begin{gathered}
\dot{r} \dot{r}+\ddot{r} q=\dot{V} \sin \eta+V \dot{\eta} \cos \eta- \\
\dot{V}_{T} \sin \eta_{T}-V_{T} \dot{\eta}_{T} \cos \eta_{T} \\
\text { Since } \dot{\eta}=(1-k) \dot{q} \quad \dot{\eta}_{T}=\dot{q}-\dot{\sigma}_{T} \quad \dot{r}=-V \cos \eta+V_{T} \cos \eta_{T}
\end{gathered}
$$


Substitute it into equation (23), we get

Where

$$
\ddot{r} \ddot{q}=-(K V \cos \eta+2 \dot{r})\left(\dot{q}-\dot{q}^{*}\right)
$$

$$
\dot{q}^{*}=\frac{V \sin \eta-V_{T} \sin \eta_{T}+V_{T} \dot{\sigma}_{T} \cos \eta_{T}}{K V \cos \eta+2 \dot{r}}
$$

We set the simulation time step to $T$ and establish the Cartesian coordinate system. We set the initial position of the missile to be the origin and put the missile and the target in the same plane. The position of the missile at $k T$ is expressed by $m x$ and $m y$. The position of the target at $k T$ is represented by $t x$ and $t y$. The motion of both the missile and the target is uniform motion and the speed are represented by $V_{m}$ and $V_{t}$. The reference direction is the initial direction of the target line-of-sight angle. $\eta_{m}$ is the angle between the flight direction of the missile and line-of-sight of the target. $\eta_{t}$ is the angle between flight direction of the target and the line-of-sight of the target. The relative distance between these two is set to $r$, and $r=$ $10000 \mathrm{~m}$. We assume that the target is traveling along a straight line and the velocity of the target is $V_{t}=420 \mathrm{~m} / \mathrm{s}$. The velocity of the missile is $V_{m}=800 \mathrm{~m} / \mathrm{s}$.

\section{B Effect of Proportional Coefficient}

We generated the simulation with the initial value $\eta_{m}(0)=\pi / 4$ and $\eta_{\tau}(0)=\pi / 4$. We set the $K$ value to be 3, 4, and 5. The trajectories of the missile and the target are shown in Figure 8 .

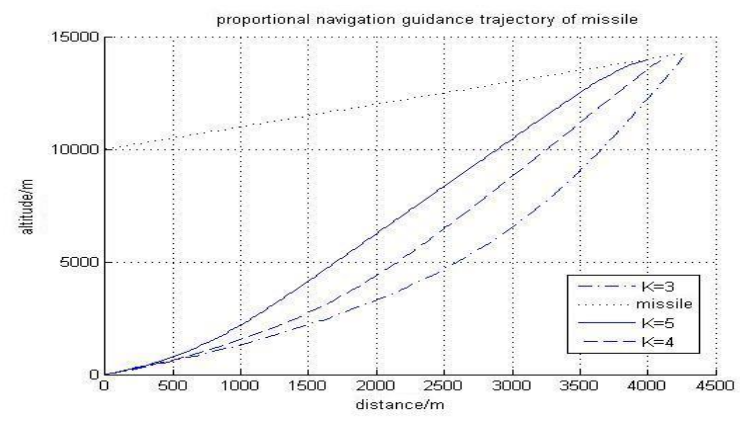

Figure 8. Simulation of the Trajectories for Different $K$ Values

As the value of $K$ becomes larger, the guidance trajectory becomes straighter. According to the Figure 8, if the $K$ value is relative small, the hitting time is shorter. If the $K$ value is relative larger, the change rate of trajectory and the necessary normal overload become smaller. The relationship between the $K$ value and encountering time is shown in Table 1.

According to the Table 1: When the $K$ value has increased to a certain value, the $t$ is not going to decrease but increase, and the trajectory is straighter.

In general, the $K$ value should be in the range from 3 to 6 . The $K$ value should not only be based on the velocity of the target, but also the firing range. In order to maximum the maneuvering capability of the missile, we need to curve the forepart of the trajectory to enhance the maneuvering capability of the missile. The posterior segment of the trajectory needs to be relatively flat. By selecting the parameters properly, we can ensure the necessary 
over-load is always less than the useable over-load. Therefore, a comprehensive attack can be finished.

\section{Effect of the Angle}

The normal over-load changes as the line-of-sight angular velocity changing since the normal over-load is directly affected by the line-of-sight angular velocity. We assume $K=5$ and take the line-of-sight of the target as the reference, the angle between the flight direction of the missile and the reference is represented by $\eta_{m}$. The angle between the flight direction and the reference is $\eta_{t}$. We generate the simulation based on the initial value $\eta_{m}=\pi / 2$, $\eta_{m}=\pi / 6, \eta_{m}=\pi / 15$, and $\eta_{\tau}=\pi / 4$. The simulation of trajectories of different line-of-sight angles is shown in Figure 9.

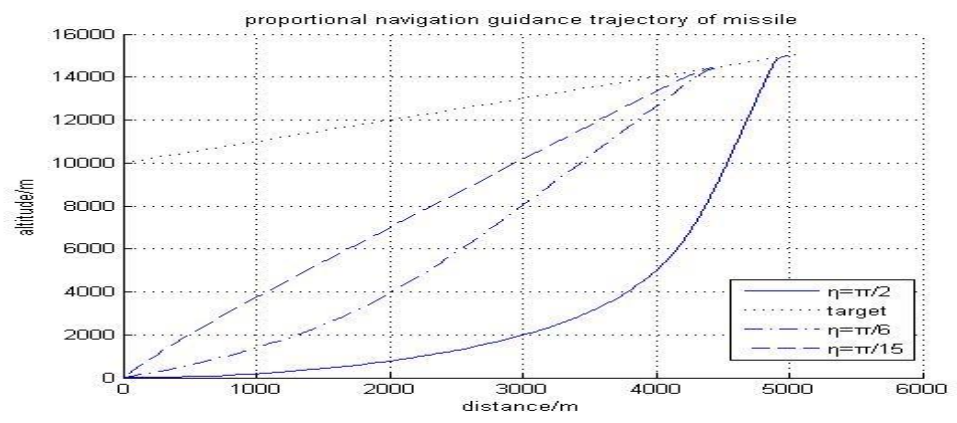

Figure 9. Simulation of the Trajectories of Different Line-of-sight Angles

Table 1. $K$ Value and Encountering Time $t$

\begin{tabular}{llllll}
\hline$K$ & 1.0 & 3.0 & 5.0 & 9.0 & 10.0 \\
\hline$t / \mathrm{s}$ & 17.9 & 15.3 & 15.1 & 15.0 & 16.3 \\
\hline
\end{tabular}

According to the Figure 9, the change rate of the trajectories is different for different $\eta_{m}$ values. When $\eta_{m}=\pi / 2$, the trajectory is more bending and the hitting time is longest. The change rate of the trajectory reaches its minimum when $\eta_{m}=\pi / 6$. The hitting time reaches its minimum when $\eta_{m}=\pi / 15$. The normal over-load directly affects the change rate of the trajectory. The greater the necessary normal over-load is, the faster the deflection of the trajectory is.

In some defined range, when $V_{m}$ increases, the encountering time $t$ decreases; when $K$ increases, $t$ decreases slowly. The velocity of missile $V_{m}$ has a stronger effect on t. when $V_{m}$ increases, the trajectory curvature $\rho$ decreases; while as the $V_{t}$ increasing, $\rho$ increases and the trajectory become more bending.

The force and the resulting acceleration during the flight of the missile can be measured by over-load. The so-called over-load $n$ refers to ratio between the resultant force of all the external forces except gravity $N$ (control force) and the weight $G$ of the missile. Over-load is a vector which has the same direction as the control force $N$. The projection of the over-load vector in the direction perpendicular to the velocity is called normal over-load. 
Figure 10 shows the relationship between normal over-load and the time $t$ for different values of the proportional coefficient $K$. According to the figure 4, the greater the guidance coefficient $K$ is, the greater amplitude of the vibration of the over-load is, the earlier the appearance of the vibration is and the longer the hitting time is. When $K=3$ and the missile is approaching to the target, the appearance time of the vibration of the normal over-load reaches its maximum.

\section{Conclusions}

In this paper, based on the traditional dynamic differential equation, an extended proportional guidance law is presented. For the sinusoidal orbital transfer motion of target after perception of danger distance, the effect of normal overload and trajectory curvature is analyzed using the extended proportional guidance law by simulation. Compared with traditional PNG laws, simulation results show that the intercept of the target based on extended proportional guidance law under the proper random quantity, when the missile is in close proximity to the target the normal overload fluctuation is improved.

The results show that the curvature radius of the trajectory is strongly affected by the proportional guidance coefficient. If change rate of trajectory angle with $t$ is small enough and the curvature radius is reasonable, the adaptability and stability of the trajectory system will improve and the hitting accuracy will also be enhanced. Proportional navigation guidance law has a good performance with smaller instruction over-load and smaller variation range of the attack angle. However, the disadvantage of the proportional navigation guidance law is that the necessary normal over-load is strongly affected by the direction of the velocity of the missile. Proportional navigation guidance law is closely related with trajectory properties, kinematic parameters and necessary normal over-load. In this simulation, we simplify the problem by ignoring the actual factors so that a certain level of error is expected. The situation in practical requires more in-depth analysis and research.

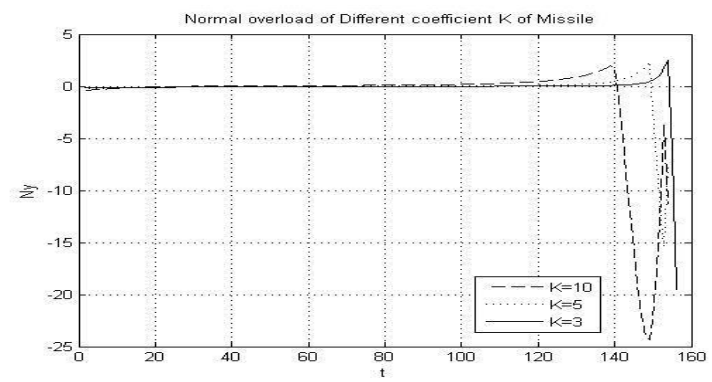

\section{Figure 10. Simulation of the Relationship between Normal Over-load and the $K$ Value}

\section{References}

[1] G. Shang, "Simulation on missile ideal trajectory under on proportion guide method", Computer Engineering and Design, (2003), vol. 24, no. 4.

[2] D. C. Quach, S. Huang, Q. Yin and C. Zhou, "An improved Direct Adaptive Fuzzy controller for an uncertain DC Motor Speed Control System”, TELKOMNIKA Indonesian Journal of Electrical Engineering, vol. 11, no. 2, (2013) February.

[3] G. Zhang, L. Zhang and Z. Chang, "Design of A Center Deviation Adaptive Bearing Pressure Assembly Machine", TELKOMNIKA Indonesian Journal of Electrical Engineering, vol. 10, no. 4, (2012) August. 
[4] M. Mehrandezh, "Robotic interception of moving objects using an augmented ideal proportional navigation guid-ance technique", IEEE Transactions on Systems, Man, and Cybernetics, (2000), vol. 30, no. 3, pp. 231244.

[5] Y. C. Sim, S B Leng and V. Subramaniam, "An all-aspect near-optimal guidance law", Dynamics and Control, vol. 10, no. 2, (2000), pp. 165-177.

[6] Z. Zhang, Y. Tong and W. Zhang, "Research on the Proportional Guidance Trajectory Simulation", Tactical Missile Technology, vol. 2, (2005), pp. 56-59.

[7] J. Zhou, Y. Tong and L. Zhang, "Simulation and Analysis of Three-dimensional Trajectory on Typical Guide Method", Ship Electronic Engineering, vol. 2, (2008), pp. 110-112.

[8] Y. Li and W. Yang, "Research on Three-dimensional Missile Trajectory Modeling Based on Proportion Guide", Command Control \& Simulation, vol. 31, no. 2, (2009), pp. 45-47.

[9] R. M. Shoucri, "Elliptical Orbit with Variable Angular Momentum", Journal of Guidance, Control, and Dynamics, vol. 18, no. 5, (1995), pp. 1213-1215.

[10] J.-W. Zhang, S.-C. Huan and Z.-C. Han, "Proportional Guidance Trajectory Based on MATLAB simulation analysis", Tactical Missile Technology, vol. 16, no. 3, (2009), pp. 60-64.

[11] E. V. C Sekhara Rao and P. V. N. Prasad, "Analysis of PMH Stepper Motor Using PDETOOL Simulations of Matlab for Different Topoligies”, TELKOMNIKA Indonesian Journal of Electrical Engineering, vol. 10, no. 4, (2012) August.

[12] G. Shang, "Proportional Navigation ideal trajectory simulation", Computer Engineering and Design, vol. 24, no. 8, (2003), pp. 66-67.

[13] Y.-F. Wang, Y.-W. Fang and X.-B. Zhou, "Proportional Navigation Research and Development", Fire Control and Command Control, (2007), vol. 32, no. 10, pp. 8-11. 